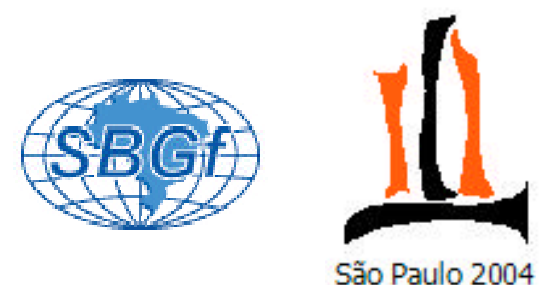

\title{
Modelamento do Sinal Gravimétrico e Aeromagnético de Alcalinas da Província Rio Verde - Iporá
}

Yára R. Marangoni, Renato F. Moraes, Maximiliano Simão, Alanna C. Dutra, Brena L. Machado e Clarino D. Vieira IAG-USP

Copyright 2004, SBGf - Sociedade Brasileira de Geofísica

Este texto foi preparado para a apresentação no I Simpósio de Geofísica da Sociedade Brasileira de Geofísica, São Paulo, 26-28 de setembro de 2004. Seu conteúdo foi revisado pela Comissão Tecno-científica do I SR-SBGf mas não necessariamente representa a opinião da SBGf ou de seus associados. E proibida a reprodução total ou parcial deste material para propósitos comerciais sem prévia autorização da SBGf.

\section{Resumo}

As alcalinas da Província Alcalina Rio Verde - Iporá, da borda norte da Bacia do Paraná possuem forte assinatura aeromagnética e gravimétrica, apresentando-se como anomalias quase circulares. As anomalias gravimétricas variam de ?15 a ?45 mGal com relação ao campo gravimétrico regional, enquanto que as anomalias aeromagneticas são da ordem de $8.000 \mathrm{nT}$. O modelamento direto usando a técnica de $2.5 \mathrm{D}$ indica que as rochas devem ser aflorantes (ou quase), variando de 5 a $15 \mathrm{~km}$ de profundidade, com circunferência média em torno de $10 \mathrm{~km}$. O sinal aeromagnético parece ser gerado por zonas quase verticais, dentro do corpo modelado pelo sinal gravimétrico, contendo mais material magnético. Estas zonas apresentam profundidades compatíveis com os modelos gravimétricos, mas espessuras da ordem de 2 a $4 \mathrm{~km}$. A inversão dos dados gravimétricos sugere profundidade menor para a base dos corpos, da ordem de $7,5 \mathrm{~km}$ e diâmetros compatíveis com o modelo direto.

\section{Introdução}

As bordas da Bacia do Paraná são caracterizadas pela presença de intrusões alcalinas de tamanho e composição variada. Na borda norte, dentro do Estado de Goiás, encontram-se duas províncias alcalinas: 1) Província Rio Verde - Iporá, no sudoeste, e 2) Província do Alto Paranaíba, mais a leste onde ocorre as alcalinas de Catalão (GO) e o maior número de ocorrências encontra-se no Estado de Minas Gerais (Ulbrich e Gomes 1981, Gomes et al. 1990). As ocorrências da Província Rio Verde - Iporá apresentam idades entre 90 e $75 \mathrm{Ma}$ (Morbidelli et al. 1995). Gibson et al. (1995) reavaliaram as datações K-Ar na província de Iporá e sugerem o intervalo de 90-80 Ma.

As alcalinas da Província Rio Verde - Iporá possuem forte assinatura aeromagnética e gravimétrica, como pode ser observado no mapa gravimétrico de Marangoni et al. (1995), e no mapa aeromagnético do Projeto Iporá (Brasil, 1974). Elas se destacam como anomalias quase circulares, sugerindo corpos com geometria cilíndrica, o que está de acordo com Gomes et al. (1990), que ressaltam que "Carbonatites occur as massive cores (plugs, stocks) usually displaying oval to circular outlines in plain view...". As anomalias gravimétricas variam de
?15 a ?45 mGal com relação ao campo gravimétrico regional (Fig. 1). As anomalias magnetométricas são da ordem de $8.000 \mathrm{nT}$ caracterizadas por um forte pico positivo e um pico negativo menor (Fig. 2). Segundo Morbidelli et al. (1995), lineamentos NW e NNW, ativos desde o Pré-Cambriano, parecem ser os responsáveis pela colocação dos complexos das províncias do Alto Paranaíba e Rio Verde - Iporá. Esses lineamentos podem ser os responsáveis pelos alinhamentos NW-SE e NESW das anomalias gravimétricas e magnéticas de algumas das alcalinas a partir da anomalia de Registro Araguaia.

Santa Fé já teve exploração de Níquel. A caracterização geofísica dessas rochas assume importância nos dias atuais em função da possibilidade de exploração de Níquel nesses corpos. Alguns desses corpos, embora apresentem forte anomalia gravimétrica e aeromagnética não afloram, e indicar possíveis profundidade do topo e largura desses corpos tem interesse econômico.

\section{Metodologia}

Os dados do levantamento aeromagnetométrico são de 1972 e 1973 e encontram-se na forma de mapas de campo total, na escala de 1:10.000, e mapas interpretativos na escala de 1:250.000. Nesses mapas foi removido um valor constante de $17.000 \mathrm{nT}$. Esses mapas foram todos digitalizados, com espaçamento de $1 \mathrm{~km}$, sendo possível obter-se os mapas de contorno digitais da área, sem que fosse feito uma nova regularização dos dados. A partir desses dados, removeu-se o IGRF para o ano de 1973.0 e um mapa residual, ressaltando as anomalias positivas e negativas (Fig.2) pode ser gerado.

Os dados gravimétricos (Fig. 1) foram obtidos em várias campanhas. Apresentam precisão variável dependendo do ano de coleta. Os anteriores a 1990, têm erros da ordem de ?0,5 mGal. Os mais recentes (maio/junho de 2004) têm erro de ?0,1 mGal, dado que o posicionamento horizontal e vertical foi feito com um GPS de duas freqüências. Os dados foram reduzido usando o valor de $2,67 \mathrm{~g} / \mathrm{cm}^{3}$ para a densidade na correção de Bouguer e a Fórmula Internacional da Gravidade de 1967 para o cálculo da gravidade normal. As estações são referenciadas às estações de $1^{\text {a }}$ ordem da RGFB (Escobar 1980) e uma rede local, de $2^{\mathrm{a}}$ ordem, implantada em uma operação conjunta OBSIS/UnB IAG/USP, com dois gravímetros e circuitos apropriados, em 1986.

A partir dos mapas de anomalias foram escolhidos perfis que cortassem as anomalias mais significativas para 0 modelamento direto e inverso. Para cada anomalia de interesse foram escolhidos dois perfis gravimétricos 
perpendiculares entre si, para melhor definir a forma geométrica do corpo (o modelamento usa geometria 2,5 $\mathrm{D}$ enquanto os corpos apresentam clara geometria 3D), sendo que um apresenta orientação NS e é coincidente com um perfil aeromagnético. Os perfis gravimétricos foram retirados usando os dados observados sendo os buracos preenchidos com dados interpolados do mapa Bouguer. Os perfis aeromagnéticos foram retirados dos mapas em papel, na escala de 1:10.000.

A modelagem conjunta 2,5D foi feita utilizando o método de Talwani et al. (1959), através do programa GravMag (Pedley et al. 1993). Um aspecto interessante deste programa é a possibilidade de ajustar os modelos à topografia do corpo aflorante, inserindo os dados de altitude ao conjunto de dados. O programa de inversão utilizado foi o Compac (Silva et al. 2001, Last e Kubik 1983) que usa os vínculos de compacidade, proximidade absoluta e desigualdade.

\section{Resultados}

No mapa de anomalias magnética e de Bouguer (Fig. 2) pode-se perceber a relação entre as anomalias gravimétricas positivas e a presença das rochas alcalinas.

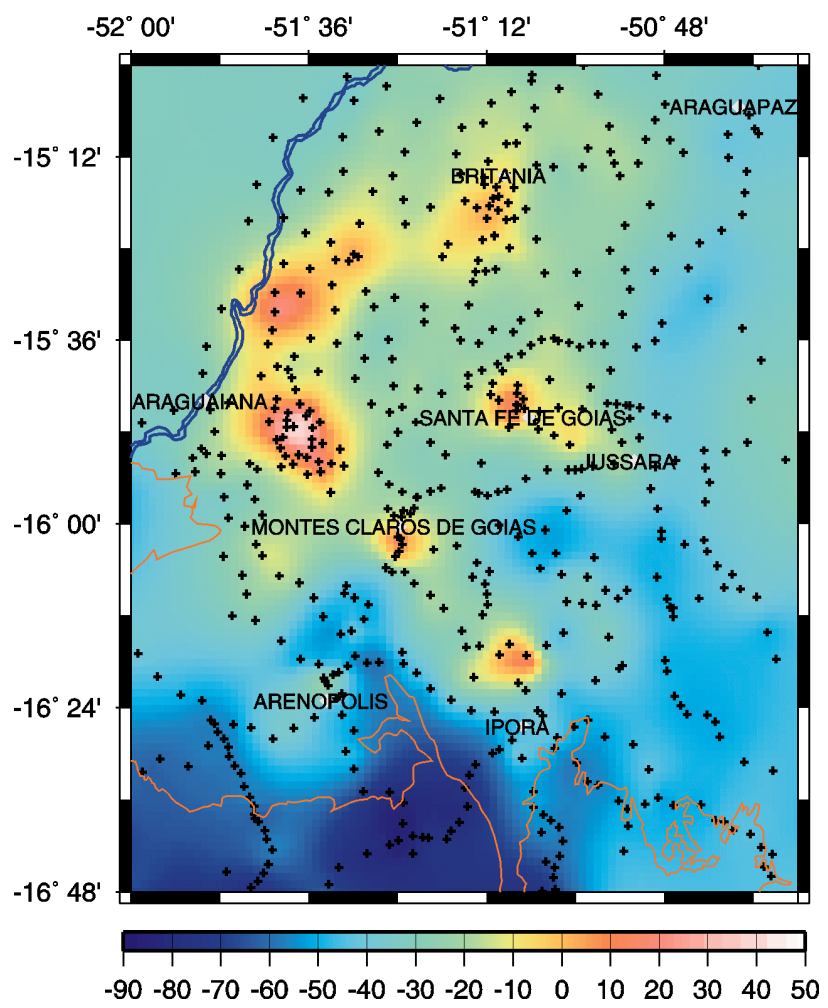

Figura 1. Mapa de anomalia Bouguer, em $\mathrm{mGal}$, com as estações gravimétricas. O contorno da Bacia do Paraná está em vermelho e o Rio Araguaia em azul.

A anomalia gravimétrica na cidade de Britânia (Fig. 1) não está associada a nenhuma anomalia magnética (Fig.
2). Um modelo para essa feição e sua correlação com rochas alcalinas será objeto de estudo. As anomalias magnéticas de Rio dos Bois e de Buriti apresentam-se individualizadas (Fig. 2) mas o correspondente gravimétrico não ocorre, sendo que o sinal dos dois corpos se misturam (Fig. 1).

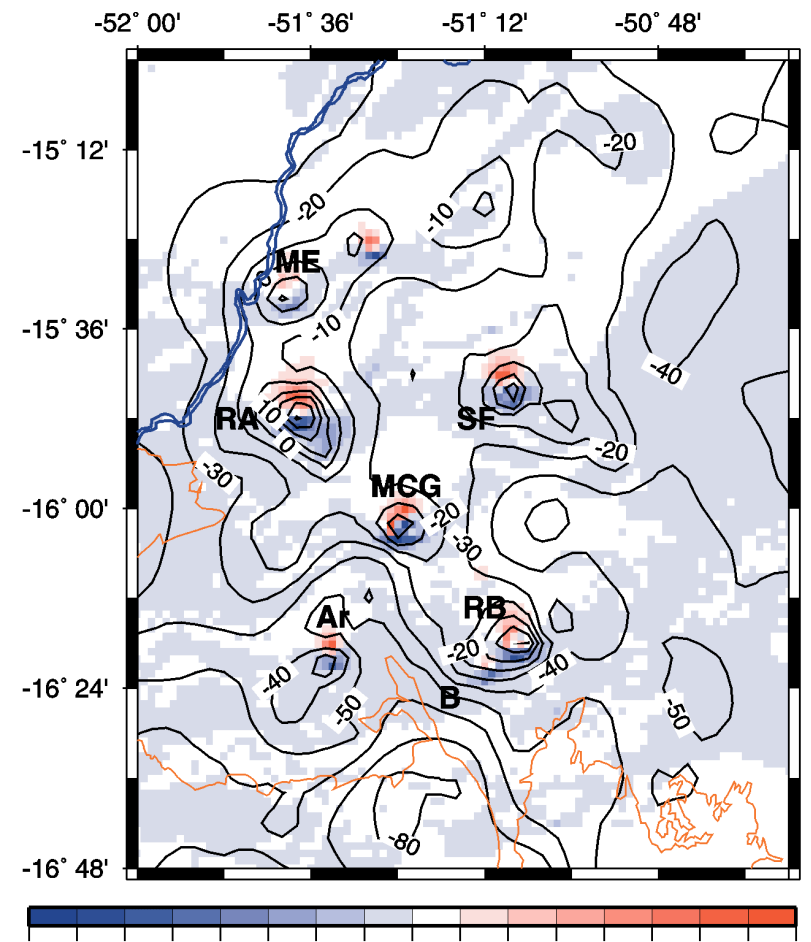

-4.0-3.5-3.0-2.5-2.0-1.5-1.0-0.5 0.0 0.5 1.01 .52 .02 .53 .03 .54 .0

Figura 2. Mapa de anomalia aeromagnéticas, escala em $10^{3} \mathrm{nT}$. Linhas pretas são os contornos de anomalia Bouguer com intervalo de $10 \mathrm{mGal}$. O contorno da Bacia do Paraná está em vermelho e o Rio Araguaia em azul. As localizações das alcalinas da Província Iporá são: MEMorro do Engenho, RA- Registro Araguaia, SF- Santa Fé, MCG-Montes Claros de Goiás, Ar- Arenópolis, RB-Rio dos Bois e B- Buriti.

O modelamento direto do sinal gravimétrico, usando a técnica de 2,5D (Pedley et al. 1993) indica que as rochas devem ser aflorantes (ou quase em alguns casos, como Registro Araguaia e anomalia a norte de Morro do Engenho), e com profundidade variando de 5 a $15 \mathrm{~km}$ e circunferência média em torno de $10 \mathrm{~km}$. O sinal aeromagnético parece ser gerado por zonas quase verticais, dentro do corpo modelado pelo sinal gravimétrico, contendo mais material magnético. Estas zonas apresentam profundidades compatíveis com os modelos gravimétricos, mas espessuras da ordem de 2 a $4 \mathrm{~km}$, além de susceptibilidade magnética variável. Um exemplo de modelamento direto pode ser visto na Fig. 3, para um perfil NS, cortando a anomalia de Montes Claros de Goiás. Essa figura ressalta a variabilidade do sinal aeromagnético em contraste com o sinal gravimétrico. Observe que o valor da susceptibilidade magnética não varia muito entre as várias faixas, estando em torno de 0,55 unidades SI. Esse perfil é representativo dos outros corpos alcalinos. 

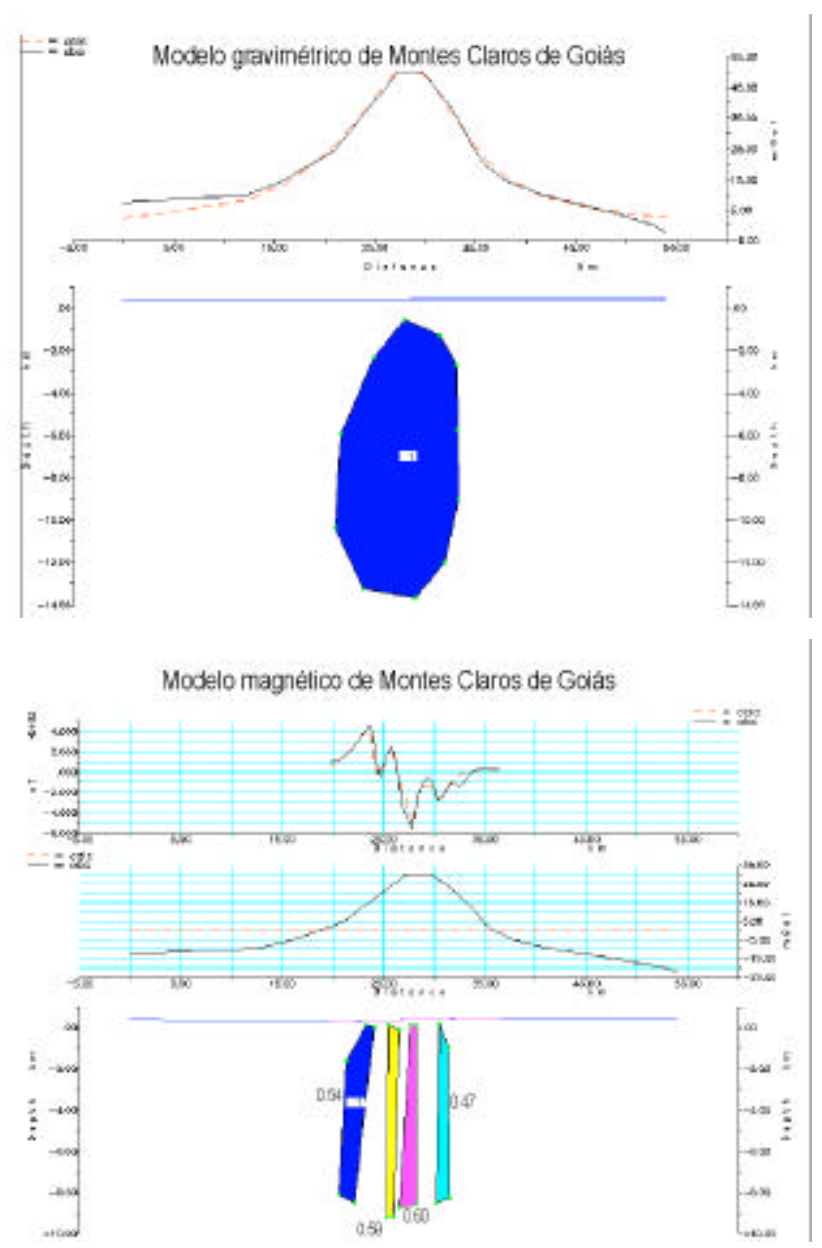

Figura 3. Modelo direto 2,5 D de um perfil gravimétrico e aeromagnético da anomalia de Montes Claros de Goiás. $O$ contraste de densidade usado foi de $0,25 \mathrm{~g} / \mathrm{cm}^{3}$. Os valores de susceptibilidade magnética, em unidades SI, encontram-se na figura.

Para o corpo de Registro Araguaia, a maior anomalia gravimétrica e aeromagnetica do conjunto e não aflorante, foi possível modelar os dois dados geofísicos em conjunto, como pode ser visto na Fig. 4.

A inversão dos dados gravimétricos sugere profundidade menor para a base dos corpos, da ordem de $7 \mathrm{~km}$ e diâmetros um pouco menores, em torno de $7,5 \mathrm{~km}$. Um exemplo para a anomalia de Registro Araguaia é mostrado na Fig. 5. Neste modelo o vínculo de compacidade mostra-se bastante adequado fornecendo uma distribuição de densidade ao redor do centro de massa do mesmo.

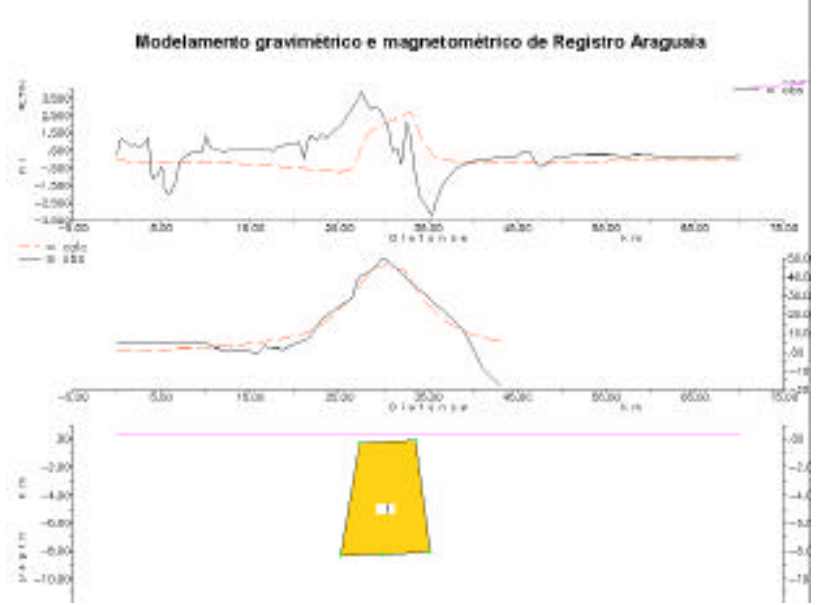

Figura 4. Modelamento conjunto do sinal gravimétrico e aeromagnético para um perfil NS cortando a anomalia de Registro Araguaia, RA na Fig. 2.

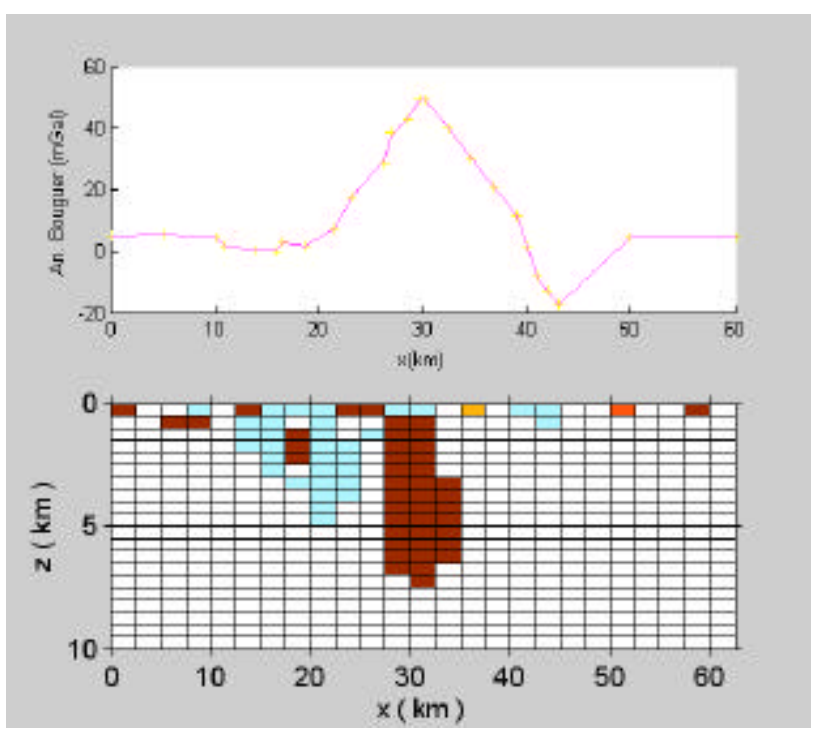

Figura 5. Inversão gravimétrica de um perfil NS cortando a anomalia de Registro Araguaia, RA na Fig. 2.

\section{Discussão e Conclusões}

Como pode ser visto nos mapas gravimétricos e aeromagnéticos as rochas das alcalinas da Província Rio Verde - Iporá apresentam forte assinatura geofísica.

O modelamento preliminar desses corpos foi feito com os dados anteriores a 2004 e novos perfis mais completos srão usados para o modelamento final. Para compensar a distribuição irregular dos dados, nos perfis gravimétricos, usou-se também os contornos do mapa Bouguer. Apesar da distribuição mais irregular dos pontos, pode-se obter corpos tridimensionais alojados na crosta superior, sendo aflorantes ou muito próximos à superfície. A fonte geradora desse magma parece ter 
concentrado os minerais magnéticos em dutos distribuídos ao redor do centro de massa do corpo.

A inversão dos dados gravimétricos e magnetométricos usando vínculos de compacidade mostrou-se apropriada, e os resultados das medidas das propriedades físicas de amostras de afloramentos das alcalinas deverá melhorar os resultados preliminares obtidos.

A caracterização geofísica desses corpos assume importância nos dias atuais em função da possibilidade de exploração de Níquel na área.

\section{Agradecimentos}

Este trabalho foi realizado com o auxílio pesquisa da Fapesp n. 2003/05088-1. Agradecemos aos geólogos Guilherme S. Canha e Paulo I. de Brito da Teck Cominco Brasil S.A. pelo auxílio na coleta de amostras das alcalinas.

\section{Referências}

Brasil, Depto. Nac. Prod. Min., 1974. Mapa de Interpretação Magnética, Projeto Iporá, escala 1:250.000. Prospec S.A.

Escobar, I. P., 1980. Métodos de Levantamentos e Ajustamento de Observações Gravimétricas Visando a Implantação da Rede Gravimétrica Fundamental Brasileira. Rio de Janeiro, ON, 57 p. (Publicação 1).

Gibson, S. A., Thompson, R. N., Leonardos, O. H., Dickin, A. P. \& Mitchell, J. G., 1995. The Late Creatceous impact of the Trindade mantle plume: evidence from large-volume, mafic, potassic magmatism in SE Brazil. J. Petrol., 36: 189-229.

Gomes, C. B., Ruberti, E. \& Morbidelli, L., 1990. Carbonatite complexes from Brazil: a review. J. South Am. Earth Sci., 3: 51-63.

Last, B. J. \& Kubik, K., 1983. Compact gravity inversion. Geophysics, 48: 713-721.

Marangoni, Y. R., Assumpção, M. \& Fernandes, E. P., 1995. Gravimetria no Norte de Goiás, Brasil. Rev. Bras. Geof., 13: 205-219.

Morbidelli, L., Gomes, C. B., Beccaluva, L., Brotzu, P., Conte, A. M., Ruberti, E. \& Traversa, G., 1995. Mineralogical, petrological and geochemical aspects of alkaline and alkaline-carbonatite associations from Brazil. Earth Sci. Rev., 39: 135-168.

Pedley, R. C., Busby, J. P. \& Dabek, Z. K. ,1993. GRAVMAG User Manual - Interactive 2.5D Gravity \& Magnetic Modelling. Britsh Geological Survey, Tecnical Report WK/93/26/R, 73p.

Silva, J. B. C., Medeiros, W. E. \& Barbosa, V. C. F., 2001. Potential-field inversion: Choosing the appropriate techique to solve a geological problem. Geophysics, 66: 511-520.
Talwani, M., Worzel, J. L. \& Landisman, M., 1959. Rapid gravity computations for two dimensional bodies with application to the Mendoncino submarine fracture zone. J. Geophys. Res., 64: 49-59.

Ulbrich, H. H. G. J. \& Gomes, C. B., 1981. Alkaline rocks from continental Brazil. Earth Sci. Rev., 17: 135-154. 